\title{
Diffraction Unlimited All-Optical Recording of Electron Spin Resonances
}

\author{
Dominik Wildanger, ${ }^{1}$ Jeronimo R. Maze, ${ }^{2}$ and Stefan W. Hell ${ }^{1, *}$ \\ ${ }^{1}$ Department of NanoBiophotonics, Max Planck Institute for Biophysical Chemistry, Am Fassberg 11, 37077 Göttingen, Germany \\ ${ }^{2}$ Facultad de Física, Pontificia Universidad Católica de Chile, Casilla 306, Santiago, Chile \\ (Received 7 April 2011; revised manuscript received 13 May 2011; published 1 July 2011)
}

\begin{abstract}
We show stimulated emission depletion microscopy to break the diffraction limit in the all-far-fieldoptical detection of magnetic fields and resonances. Electron spin resonances from single nitrogenvacancy centers in diamond located at subdiffraction proximities are fully discerned. Since diffraction is overcome by disallowing the signaling state through an optical transition such as stimulated emission, the spin state remains unaffected and amenable to microwave manipulation. Stimulated emission depletion presents a universal scheme for superresolving spin resonances detectable by fluorescence.
\end{abstract}

Optical detection of magnetic resonances (ODMR) provides convenient access to spin states and magnetic fields, which is why optical spin readouts have been harnessed in rather diverse areas of research, such as quantum computation $[1,2]$, atomic clocks, and magnetometry $[3,4]$. The electron spin associated with charged nitrogen-vacancy (NV) centers in diamond is optically addressable [5], because it can be polarized via an optical excitation, while its orientation can be inferred from the emitted fluorescence [6,7]. Another feature of NV spin states in isotopically purified diamond is their $>1 \mathrm{~ms}$ coherence time at room temperature [8], which renders them most promising as qubits for scalable quantum computers and atom-sized alloptical sensors of magnetic fields [9]. However, when reading out or manipulating these and other optically addressable spins with focused light, the obtainable spatial resolution is limited to about $\lambda /(2 n \sin \alpha) \approx 200 \mathrm{~nm}$, with $\lambda$ and $n \sin \alpha$ denoting the wavelength of light and the numerical aperture of the lens, respectively. In fact, the resolution of all ODMR experiments performed with conventional optics is limited by diffraction.

Recently, concepts [10] have been developed to overcome the diffraction barrier in far-field fluorescence microscopy, ultimately providing a resolution down to a few nanometers. A first example is stimulated emission depletion (STED) microscopy [11], which overcomes the diffraction barrier by sequentially turning off the fluorescence of features closer than $\lambda /(2 n \sin \alpha)$, such that they fluoresce consecutively from more sharply defined coordinates in space. By resolving individual electron spin resonances (ESRs) of densely packed NV centers in diamond, we now overcome the diffraction barrier in the optical detection of magnetic resonances and fields. In particular, we show that STED microscopy resolves spins basically without a diffraction limit. A fundamental advantage of STED over a recent technique called spin-reversible saturable optical fluorescence transitions [12] demonstrated with $\mathrm{NV}$ centers is that no microwave-induced changes in spin orientation are needed and no prior knowledge of the resonance behavior of the spins is required. The diffraction barrier is overcome just by exploiting optical transitions between a fluorescent and a nonfluorescent electronic state. Consequently, (i) the recording is virtually backgroundfree and in the case of densely packed NV centers accelerated up to $10^{4}$-fold, (ii) the spin orientation is unaffected, meaning that any ODMR sequence can be applied, and (iii) the method is not limited to NV centers but is also applicable to other systems with optically readable spins.

Nonetheless, the spin associated with charged NV centers in diamond is nearly perfect for demonstrating the principles of STED-ODMR. The two unpaired electrons of the NV center (a substitutional nitrogen next to a carbon vacancy) form a spin triplet in the ground state ${ }^{3} A_{2}$ with sublevels $m_{s}=0$ and $\left|m_{s}\right|=1$. Without external field, the $\left|m_{s}\right|=1$ states are degenerate and separated by $2.87 \mathrm{GHz}$ from the $m_{s}=0$ state [13]. The $m_{s}$ can be optically probed, because $m_{s}=0$ displays a higher fluorescence probability as compared to the $\left|m_{s}\right|=1$ states [7], because upon optical excitation ${ }^{3} A_{2} \rightarrow{ }^{3} E$, the $\left|m_{s}\right|=1$ states exhibit an $\approx 30 \%$ reduced fluorescence caused by the increased probability to cross nonradiatively from the fluorescent state ${ }^{3} E$ (presumably) to the metastable singlet state ${ }^{1} A_{1}$ and finally to the ground state ${ }^{3} A_{2}[6,14]$. Importantly, this also polarizes the spin to $m_{s}=0$.

A magnetic field splits the $\left|m_{s}\right|=1$ states in the first approximation proportional to the projection of the field onto the axis of the NV center (Zeeman effect), giving a split resonance with a frequency difference $\Delta f=$ $2 g \mu_{B} B \cos \theta$, with $g$ and $\mu_{B}$ denoting the $g$ factor and the Bohr magneton, respectively; $\theta$ is the angle between the field vector and the NV axis [13]. Hence, if the angle is known, the ESR spectrum reveals the local magnetic field [3].

In essence, STED ensures that features (here NV) residing closer than $\lambda /(2 n \sin \alpha)$ of optical excitation $\left({ }^{3} A_{2} \rightarrow{ }^{3} E\right)$ cannot signal in parallel but only sequentially [15]. For this reason, they are successively deprived of their fluorescence ability by using a beam of light, called the 
STED beam, which keeps the NV dark by prohibiting them to occupy the fluorescent state ${ }^{3} E$. Features experiencing a STED beam intensity $I>I_{s}$ remain dark even when exposed to the excitation light; $I_{s}$ is the intensity at which the ${ }^{3} E$ occupation is reduced to $1 / e$. Stimulated emission can be substituted by any other optical process that reversibly diverts the NV from the ${ }^{3} E$ [10]. The STED beam features a narrow wavelength range $(\sim 775 \mathrm{~nm})$ at the red edge of the emission spectrum (see Fig. 1), such that stray light can be excluded by detecting between 630 and $720 \mathrm{~nm}$. Importantly, its intensity vanishes at a controllable point where the emitters can still assume the ${ }^{3} E$; more precisely, the ${ }^{3} E$ is allowed in a range given by $\lambda /\left(2 n \sin \alpha \sqrt{1+I / I_{s}}\right)$ around the zero intensity point, where the intensity is $<I_{s}$. $I$ is the maximum intensity bordering the minimum. Thus, the sequential permission of ${ }^{3} E$ in a ${ }^{3} A_{2}$ neighborhood discerns the features from their nearby peers. The STED beam is prepared as a doughnut that is overlaid with a Gaussian excitation beam (Fig. 1). Images are constructed by translating the beams and hence the coordinate of ${ }^{3} E$ occupation across the sample.

The fluorescence capability of NV centers in bulk diamond can be relentlessly switched off just by applying a STED beam intensity $>I_{s} \approx 42 \mathrm{MW} / \mathrm{cm}^{2}$. STED

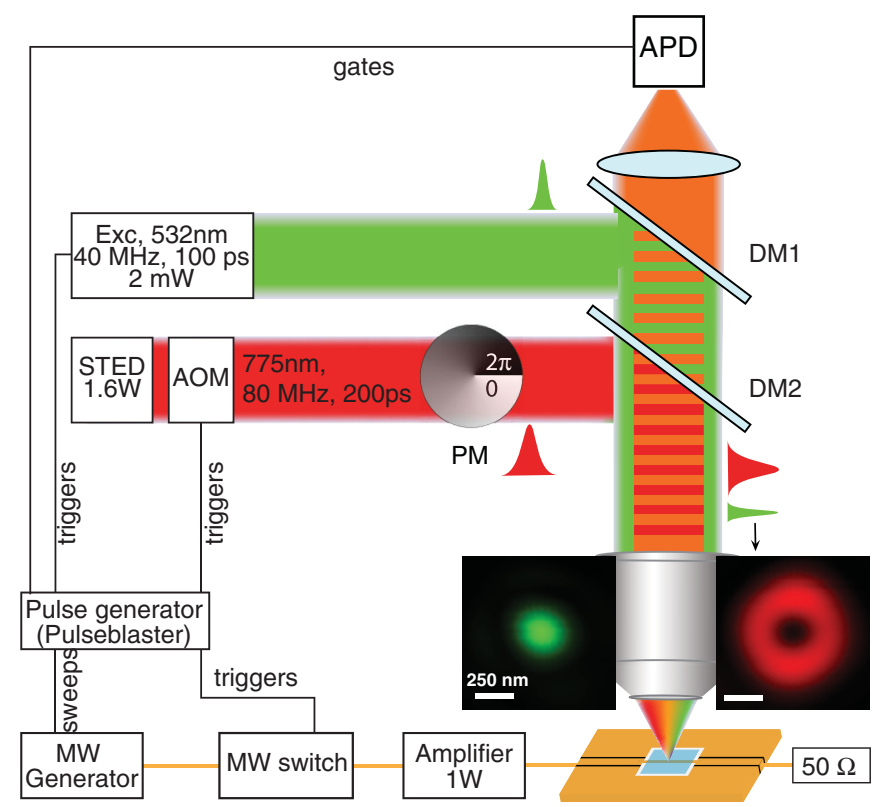

FIG. 1 (color). STED nanoscope for optical detection of magnetic resonances of NV centers. Excitation by a pulsed (100 ps) laser diode, synchronized with a laser for STED, providing down-chirped pulses at $775 \mathrm{~nm}$ of $200 \mathrm{ps}$ duration; the laser intensity is modulated by an acousto-optical modulator (AOM); the STED focal doughnut is produced by imprinting a $0-2 \pi$ helical phase ramp. The overlaid beams are guided to the objective lens by using dichroic mirrors (DM1 and DM2) and focused into the sample (inset panels show focal intensity spots). Scanning is performed with a 3D piezo scanner of nanometric precision. Fluorescence collected by the lens is focused onto a confocalized detector (APD). microscopy on NV centers has displayed the highest alloptics-based resolving power so far, down to $6 \mathrm{~nm}$ [15]. Enabling both subdiffraction resolution and spin imaging, we explored the use of NV centers for all-far-field-optical nanoscopy of spin states and magnetic resonances.

We used ultrapure (electronic grade, element six) diamond crystals featuring NV centers 1-5 $\mu \mathrm{m}$ beneath the surface, created by nitrogen implantation followed by annealing [16]. Densely packed NV centers and the associated electron spin resonances were recorded both in the confocal and in the STED mode. Applying $532 \mathrm{~nm}$ light for excitation polarizes the NV centers to the bright $m_{s}=0$ state giving the fluorescence base line in all ESR traces. A constant magnetic field $\vec{B}_{0,1}$ was applied by placing a permanent magnet close to the diamond sample, lifting the degeneracy of the $\left|m_{s}\right|=1$ states by Zeeman splitting. Sweeping the microwave frequency across the $2.87 \mathrm{GHz}$ resonance identified the frequencies at which the spins were transferred from $m_{s}=0$ to $m_{s}=-1$ or to $m_{s}=1$ and back, whereby the difference in frequency between the two transitions is in proportion to the magnetic field experienced by the electrons. The resonances are dips because the $\left|m_{s}\right|=1$ states fluoresce $\sim 30 \%$ less than the $m_{s}=0$ state

Because of its diffraction-limited resolution, the confocal mode fails in separating the NV centers [Fig. 2(a)]; it renders a blurred fluorescence spot representing an unknown number of emitters. Recording an ESR spectrum at the position of maximum fluorescence exhibits four resonance lines, suggesting (at least) two NV centers of different orientation.

Adjusting $\sim 50 \mathrm{~nm}$ resolution by STED $(I \approx$ $1.6 \mathrm{GW} / \mathrm{cm}^{2}$ ) revealed five centers. The corresponding ESR traces also show marked differences to their confocal counterparts both in the depth of the resonance dips and in the number of dips observed. Differences and similarities between single NV defects can be inferred. The three magnitudes in Zeeman splitting indicate three different orientations of the centers to the applied field $\vec{B}_{0,1}$ (Fig. 2). The contrast of the STED-ESR resonances is increased $(\sim 25 \%)$ because now just a single spin signals at a time.

To demonstrate the potential of NV as magnetometers of atomic extent, we show changes in the ESR spectrum induced by turning the magnet by $90^{\circ}$ leading to a different field $\vec{B}_{0,2}$ [Fig. 2(d)]: NV having the same orientation within the crystal still display identical splitting between the $m_{s}=-1$ and $m_{s}=1$ resonance lines, but the magnitude of the split changes dramatically, proving the ability to sense changes of external magnetic fields at the nanoscale. Since the center under observation is unaffected by STED, all conventional ODMR experiments such as highsensitivity ac and dc field sensing by echo sequences and relaxation times measurements can be performed at the same speed and noise level as in the confocal mode but without being limited by diffraction. 
We note that the resolution attainable in this study was limited by the power of the STED laser which, having a higher repetition rate than in previous experiments [15], provided a lower maximum intensity $I$. Larger $I$ also call for suppressing laser light by time-gated detection. Provided with these measures, resolution in the single digit nanometer range can be achieved. However, such
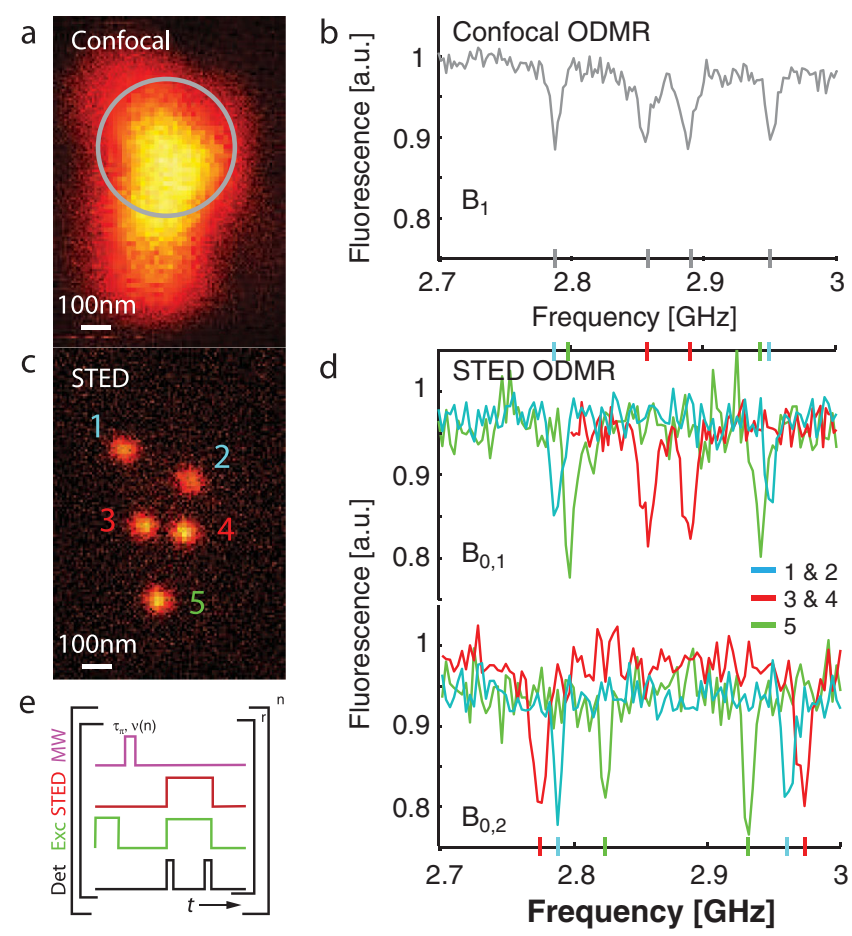

FIG. 2 (color). Superresolution far-field ODMR and ESR with Zeeman splitting induced by magnetic field $\vec{B}$. (a) Confocal image fails to resolve densely packed NV centers. (b) Confocal ESR signal acquired at the position of maximum fluorescence exhibits 4 resonance lines indicating more than one spin within the diffraction-limited area of signal origin. (c) STED image (dwell time $1 \mathrm{~ms}$ per pixel) resolves 5 spins, and (d) STED-ODMR reveals their ESR traces individually. Three spin orientations are found with respect to the crystal orientation, as indicated by the numbers in cyan $(2$ spins, splitting of $174 \mathrm{MHz}$ at $\vec{B}_{0,1}$ and $144 \mathrm{MHz}$ at $\vec{B}_{0,2}$ corresponding to local fields of 30 and $25 \mathrm{G}$, respectively), red [2 spins, splitting of $200\left(\vec{B}_{0,1}\right)$ and $30 \mathrm{MHz}\left(\vec{B}_{0,2}\right)$ ], and green [1 spin with a splitting of $108\left(\vec{B}_{0,1}\right)$ and $\left.165 \mathrm{MHz}\left(\vec{B}_{0,2}\right)\right]$. From the splitting asymmetries we derived $\left|\vec{B}_{0,1}\right|=(4.46 \pm 0.03) \mathrm{mT}$ and $\left|\vec{B}_{0,2}\right|=$ $(3.02 \pm 0.17) \mathrm{mT}$. Far-field optical magnetometry with nanometric resolution is demonstrated by recording two STEDESR traces with different magnetic fields applied $\left(\vec{B}_{0,1}\right.$ and $\vec{B}_{0,2}$ ); the ESR spectra change strongly with the field. (e) The pulsed ESR sequence. First the $\mathrm{NV}^{-}$center is initialized to the $m_{s}=0$ state by exposing it to green light. Afterwards a microwave $\pi$ pulse of varying frequency $\nu(n)$ is applied. The spin signal is read out with high spatial resolution by simultaneously illuminating the sample with excitation and STED light. The fluorescence occurring during the first $300 \mathrm{~ns}$ of exposition is recorded and compared to the reference value recorded afterwards. To increase the signal-to-noise ratio, the sequence is repeated $r$ times (typically $10^{4}$ ) for each microwave frequency. resolution was not needed here to separate the NV. Since STED imaging has been applied to nanodiamonds, STEDODMR will also be applicable to nanodiamonds acting as magnetic probes within living cells [17] at nanometric resolution.

For applications involving interacting spins, it is important to find out if the STED beam alters the spin state. By disallowing the ${ }^{3} E$ through an instant optically stimulated transition to ${ }^{3} A_{2}$, the STED beam should ideally counteract the optically induced polarization. More generally, a STED beam of intensity $\gg I_{s}$ should protect the spin $m_{s}$ from optical polarization to $m_{s}=0$.

We therefore investigated the influence of the STED beam on the Rabi oscillations between $m_{s}=0$ and $\left|m_{s}\right|=1$ by superimposing the excitation beam at $40 \mathrm{MHz}$ with a synchronized Gaussian STED beam at $80 \mathrm{MHz}$ and applied the sequence of optical and microwave pulses (operating at $2.842 \mathrm{GHz}$ ) sketched in Fig. 3(a). After initialization to $m_{s}=0$, a microwave pulse transferred the electrons to a spin state to be probed by an optical excitation pulse $(\lambda=532 \mathrm{~nm})$, which was serving as a reference. Immediately afterwards, the same sequence was applied, with the only difference being that an additional $532 \mathrm{~nm}$ pulse was applied right after the microwave pulse, disturbing the state. The optical disturbance lasted $200 \mathrm{~ns}$, which is the time required to polarize the spin back to $m_{s}=0$ by excitation to the ${ }^{3} E$. Figure 3 shows that just varying the duration $\tau$ of the microwave pulses between 0 and 300 ns delineated the Rabi oscillations, whereas upon adding the disturbation pulses the oscillations were largely destroyed. However, by overlapping the disturbing excitation pulses spatially and temporally with STED pulses (30.7 $\mathrm{mW}, \sim 1 \mathrm{GW} / \mathrm{cm}^{2}$ average intensity), the Rabi oscillations could be recovered, proving that STED can "protect" the spin population from optical destruction.
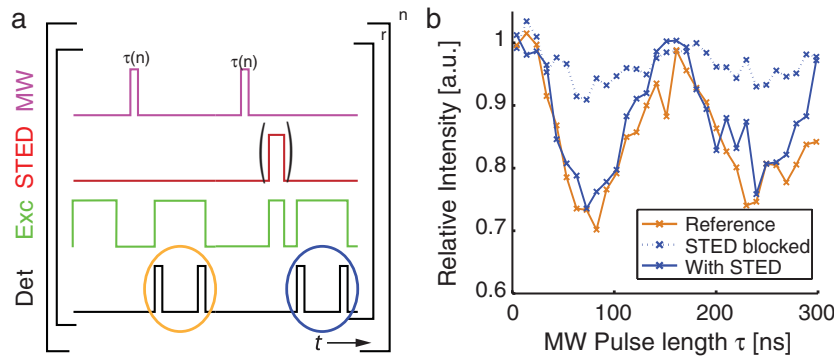

FIG. 3 (color). Preservation of spin population (Rabi oscillations) by STED. (a) Pulse sequence: (i) An undisturbed Rabi oscillation yields a reference fluorescence signal; (ii) an optical excitation pulse train (EXC, $200 \mathrm{~ns}$ exposure, $240 \mu \mathrm{W}, 532 \mathrm{~nm}$, 100 ps pulse length) succeeding the microwave pulse (MW) repolarizes the spin to $m_{s}=0$, thus disturbing the spin prepared by the microwave. (b) While the reference displays a nearly perfect Rabi oscillation (yellow), the oscillation is disturbed by adding the second excitation pulse (dotted blue line). If a STED pulse is applied jointly with the disturbing pulse, the oscillations are preserved (solid blue line). Det indicates the detection window and $n$ the number of iterations. 
The protection can be attributed to the stimulated emission ${ }^{3} E \rightarrow{ }^{3} A_{2}$. We found that the contrast of the Rabi oscillations, and hence the spin protection, increased with increasing STED beam intensity, as expected. With $I$ enabling a resolution of $\sim 100 \mathrm{~nm}$, the Rabi oscillations displayed almost full contrast. However, when increasing $I$ further, the contrast and hence the protective effect was reduced due to an increased population of a metastable dark state. While the dark state can be depopulated with a few $532 \mathrm{~nm}$ pulses of $\sim 100 \mathrm{~ns}$ duration, the spin information is lost. In Ref. [18], it is shown how the contrast of the Rabi oscillations and relative intensity (indicating an increase of the dark state population) depend on the STED beam intensity. Hahn-echo sequences have also been performed to investigate if the phase of the spin state can be protected as well (see [18]) which show that upon illumination with excitation and STED light the phase is lost. A possible explanation for the phase loss is the difference in the effective magnetic field between the ground and excited states due to differences in the zero field splitting and a difference in the coupling to external spins. Even if the NV is deexcited by stimulated emission, it has spent some time in the excited state and thus acquired a phase caused by the coupling of the excited state, meaning that the symmetry of the Hahn-echo sequence is broken. Importantly, the use of an optical transition such as stimulated emission for breaking the diffraction barrier (by onoff switching of NV signaling) leaves spin states free for microwave control. Nanoscopy concepts using other transitions, such as the various forms of ground state depletion [19-21], should also enable nanoscale resolution ODMR. Some of them should even entail lower optical intensities, as is advantageously the case for spin-reversible saturable optical fluorescence transitions [12]. However, exploiting metastable dark states, such as in recently reported ground state depletion concepts for turning off the NV signal [21], will also affect the spin states. Some of these concepts also imply a nonzero background [12,19], making it more difficult to discern densely packed centers.

While the use of metastable dark states for overcoming the diffraction barrier depends on the emitters used in the ODMR experiment, STED is a general approach that is applicable to basically any spin system whose signal can be reversibly disturbed by light. Therefore, our approach can be extended also to other spin systems involving a signaling state of finite lifetime, whose occupation can be forbidden by stimulated emission or another optically induced transition. It will also be interesting to search for other optically readable spin systems where the disallowance of the signaling state is not accompanied by a spin disturbing transition. Furthermore, a recent study investigated the effect of the dark state population on the nuclear spin [22].

So far, only STED has the potential to read out the orientation of spins closer than the diffraction barrier nondestructively, making it interesting to investigate how far nondestructive spin imaging can be pushed beyond the diffraction barrier. If spin states can be prepared consecutively and preserving the phase of adjacent spin states is not relevant, as is the case in nanoscale optical magnetometry, our results show that all-far-field optical detection of magnetic resonances and fields is possible without a diffraction limit.

We thank R. Walsworth and M. Lukin (Harvard) for motivating discussions. J. Meijer, S. Pezzagna (Rubion, Bochum), B. Naydenov (Stuttgart), and H. Schill (MPI Göttingen) are thanked for providing samples. We acknowledge discussions with E. Rittweger, K. Y. Han, C. Eggeling, and G. Balasubramanian (MPI) and thank J. Jethwa (MPI) for developing electronics. We also thank I. Tkach (MPI) for microwave simulations and A. Schönle (MPI) for the software IMSPECTOR. J. R. M. was supported by the Conicyt Fondecyt Grant No. 11100265. D. W. and S.W.H. acknowledge the German National Academic Foundation and the DFG, respectively.

*shell@gwdg.de

[1] J. Wrachtrup, Proc. Natl. Acad. Sci. U.S.A. 107, 9479 (2010).

[2] P. Neumann et al., Nature Phys. 6, 249 (2010).

[3] S. Steinert et al., Rev. Sci. Instrum. 81, 043705 (2010).

[4] J. Maze et al., Nature (London) 455, 644 (2008).

[5] A. Gruber et al., Science 276, 2012 (1997).

[6] J. Wrachtrup and F. Jelezko, J. Phys. Condens. Matter 18, S807 (2006).

[7] F. Jelezko and J. Wrachtrup, J. Phys. Condens. Matter 16, R1089 (2004).

[8] G. Balasubramanian et al., Nature Mater. 8, 383 (2009).

[9] G. Balasubramanian et al., Nature (London) 455, 648 (2008).

[10] S. W. Hell, Nat. Methods 6, 24 (2009).

[11] S. W. Hell and J. Wichmann, Opt. Lett. 19, 780 (1994).

[12] P. Maurer et al., Nature Phys. 6, 912 (2010).

[13] J. Loubser and J. Vanwyk, Rep. Prog. Phys. 41, 1201 (1978).

[14] N. B. Manson, J. P. Harrison, and M. J. Sellars, Phys. Rev. B 74, 104303 (2006).

[15] E. Rittweger, K. Y. Han, S. E. Irvine, C. Eggeling, and S. W. Hell, Nat. Photon. 3, 144 (2009).

[16] J. Meijer et al., Appl. Phys. Lett. 87, 261909 (2005).

[17] F. Neugart, A. Zappe, F. Jelezko, C. Tietz, J. Boudou, and A. Krueger, Nano Lett. 7, 3588 (2007).

[18] See supplemental material at http://link.aps.org/ supplemental/10.1103/PhysRevLett.107.017601 for Hahn echo experiments and dark state effects on spin orientation population.

[19] E. Rittweger, D. Wildanger, and S. W. Hell, Europhys. Lett. 86, 14001 (2009).

[20] S. W. Hell and M. Kroug, Appl. Phys. B 60, 495 (1995).

[21] K. Han, S. Kim, C. Eggeling, and S. Hell, Nano Lett. 10, 3199 (2010)

[22] G. Waldherr et al., Phys. Rev. Lett. 106, 157601 (2011). 